\title{
Mastitis granulomatosa: un reto radiológico
}

\section{Granulomatous mastitis: a radiological challenge}

\author{
Juan F. Barco-Manrique ${ }^{1 *}$, Natalia Jaramillo-Botero ${ }^{1}$, Miguel E. Ochoa-Vera ${ }^{2}$, Isabel Bolivar-Aguilera ${ }^{3}$ y \\ Silvia N. Vera-Campos ${ }^{4}$ \\ ${ }^{1}$ Departamento de Radiología e Imágenes Diagnosticas, Clínica FOSCAL, Floridablanca; ${ }^{2}$ Departamento de Epidemiología Clínica, Grupo de \\ Investigaciones Clínica, Clínica FOSCAL, Floridablanca; ${ }^{3}$ Departamento de Patología, Laboratorio Bolivar, FOSCAL Internacional, Floridablanca; \\ ${ }^{4}$ Departamento de Radiología e Imágenes Diagnosticas, Facultad de Ciencias de la Salud, Universidad Autónoma de Bucaramanga, Bucaramanga. \\ Colombia
}

\section{Resumen}

Introducción: La mastitis granulomatosa es una enfermedad inflamatoria crónica benigna de etiología desconocida y buen pronóstico, sin embargo, es una entidad de difícil diagnóstico debido a sus hallazgos inespecificos y variabilidad en los diagnósticos diferenciales. Objetivo: Brindar herramientas útiles en el diagnóstico oportuno de mastitis granulomatosa con sus principales hallazgos imagenológicos. Materiales y métodos: Se realizó un análisis retrospectivo de los hallazgos imagenológicos en 17 mujeres con diagnóstico histopatológico de mastitis granulomatosa entre enero del 2017 y marzo del 2019 con una revisión bibliográfica del tema. Resultados: De 17 pacientes con resultado histopatológico de mastitis granulomatosa, 15 fueron valoradas inicialmente con ultrasonografía, encontrando que el 53,3\% presentaron masa heterogénea hipoecoica, seguido de extensiones tubulares en un 46,6\%, además un 26,6\% presentó masa circunscrita, colección y adenopatías axilares hallazgos acordes con la literatura mundial. Solo a una paciente se le realizó mamografía, en la cual se encontró una asimetría focal de la densidad del tejido mamario siendo el hallazgo más frecuente. Se realizó a una paciente resonancia magnética con realce no masa y fístula cutánea como complicación, que equivale al 6,6\%. Conclusión: Nuestros hallazgos imagenológicos son concordantes con la literatura mundial y se logró identificar las características radiológicas centrados principalmente en ultrasonido.

Palabras clave: Mastitis granulomatosa. Mamografía. Ultrasonografía. Resonancia magnética. Mama. Diagnóstico.

\section{Abstract}

Introduction: Granulomatous mastitis is a benign chronic inflammatory disease of unknown etiology, and a good prognosis, however, it is an entity of difficult diagnosis due to its nonspecific findings and variability in differential diagnoses. Objective: To provide useful tools in the timely diagnosis of granulomatous mastitis with its main imaging findings. Materials and methods: We performed a retrospective analysis of the imaging findings in 17 women with histopathological diagnosis of granulomatous mastitis between January 2017 and March 2019 with a literature review of the subject. Results: Of 17 patients with a histopathological outcome of granulomatous mastitis, 15 were initially assessed with ultrasonography, finding that $53.3 \%$ had a heterogeneous hypoechoic mass followed by tubular extensions in $46.6 \%$, in addition $26.6 \%$ presented a circumscribed mass, axillary lymph node or collection. Only one patient underwent to mammography, in which a focal asymmetry of breast tissue was found. A magnetic resonance imaging was performed on a patient with no mass and

Correspondencia:

*Juan F. Barco-Manrique

E-mail: fbarcom @gmail.com
Disponible en internet: 21-09-2021

Rev Chil Radiol. 2021;27(3):127-131

www.resochradi.com

0717-9308 / @ 2020 Sociedad Chilena de Radiología. Publicado por Permanyer. Éste es un artículo open access bajo la licencia CC BY-NC-ND (https://creativecommons.org/licenses/by-nc-nd/4.0/). 
Rev Chil Radiol. 2021;27(3)

cutaneous fistula also had a fistula equivalent to $6.6 \%$ of complication. Conclusion: Our imaging findings of our patients are consistent with the literature. We have identified the radiological characteristics mainly focused on ultrasound.

Key words: Granulomatous mastitis. Mammography. Ultrasonography. MRI. Breast. Diagnosis.

\section{Introducción}

La mastitis granulomatosa es una enfermedad inflamatoria crónica benigna de etiología desconocida y generalmente es un diagnóstico de exclusión ${ }^{1-3}$.

Su presentación es amplia, siendo la manifestación clínica más común una masa dolorosa unilateral palpable de tamaño variable $(1-20 \mathrm{~cm})$ acompañado de edema cutáneo y/o eritema. También se puede presentarse en forma de abscesos con o sin drenaje a la piel y es frecuente la presencia de adenopatías axilares ${ }^{1,4}$.

El embarazo y la lactancia son los únicos factores con asociaciones bien establecidas en la mastitis granulomatosa ${ }^{1}$. No se ha encontrado asociación con antecedentes de enfermedad granulomatosa y se han descrito casos asociados a Corynebacterium kroppenstedtii ${ }^{5}$.

El diagnóstico es difícil y debe basarse en un enfoque multidisciplinario. Es importante excluir otras causas secundarias de mastitis antes de realizar un diagnóstico. Entre las patologías que descartar encontramos el carcinoma inflamatorio, infecciones crónicas, enfermedades autoinmunes, mastitis tuberculosa, infecciones por hongos o por parásitos 3 ,6-8.

Entre las complicaciones están la recurrencias, fístulas cutáneas $(6,67 \%)$ y la sobreinfección ${ }^{8}$, por lo cual es de vital importancia realizar un diagnóstico oportuno basado en la clínica, hallazgos imagenológicos e histopatología.

\section{Materiales y métodos}

Se realizó un estudio retrospectivo de los hallazgos imagenológicos en 17 mujeres con diagnóstico histopatológico de mastitis granulomatosa entre enero del 2017 y marzo del 2019 que acudieron al servicio de radiología e imágenes diagnósticas.

En esta investigación no realizó intervención alguna o modificación intencionada de las variables biológicas, fisiológicas, psicológicas o sociales de los individuos. Adicionalmente, este estudio se apega a las normas éticas elaboradas en la Declaración de Helsinki modificada (Brasil 2013) y al reporte de Belmont, teniendo en cuenta los principios bioéticos de beneficencia, justicia y respeto a los demás.

Igualmente, la institución encargada aportó los datos necesarios por medio de sus historias clínicas electrónicas, previa evaluación y aprobación por parte del comité de ética institucional.

\section{Resultados}

Se analizaron 17 pacientes, de las cuales 15 fueron valoradas inicialmente con ultrasonografía. El 53,3\% presentaron masa heterogénea hipoecoica seguido de extensiones tubulares en un $46,6 \%$, el $26,6 \%$ presentó masa circunscrita, colección y adenopatías axilares (Fig. 1); adicionalmente se encontró aumento de la señal Doppler color en el tejido circundante y/o en la lesión en un $20 \%$ de los casos.

Solo a un paciente se le realizó mamografía, en la cual se encontró una asimetría focal de la densidad del tejido mamario (Fig. 2). Se realizó a un paciente resonancia magnética con realce no masa y fístula cutánea como complicación (Fig. 3).

Los 17 pacientes tenían resultado histopatológico de mastitis granulomatosa. Se observaron infiltrados inflamatorios con linfocitos, células plasmáticas, histiocitos y neutrófilos. Se evidenciaron múltiples agrupaciones de células gigantes multinucleadas y granulomas no caseificantes (Fig. 4).

\section{Discusión}

Los hallazgos imagenológicos en la mastitis granulomatosa han sido reportados con relativa menor frecuencia en la literatura radiológica. Las características mamográficas y ecográficas se superponen con la de infección mamaria y lesiones malignas ${ }^{9,10}$.

Las estrategias para obtener imágenes de esta entidad dependen de la edad del paciente, las manifestaciones clínicas y factores de riesgo. La ecografía, mamografía y, menos frecuentemente, la resonancia magnética, han demostrado ser útiles ${ }^{11}$.

Una vez que se sospecha un diagnóstico de mastitis granulomatosa, se debe comenzar con estudios de imagen convencional; una mamografía de diagnóstico si la paciente tiene más de 40 años de edad y un ultrasonido si tiene menos de 30 años ${ }^{11}$; sin embargo, algunos autores apoyan la realización de mamografía unilateral opcional en pacientes menores de 40 años ${ }^{12}$. 


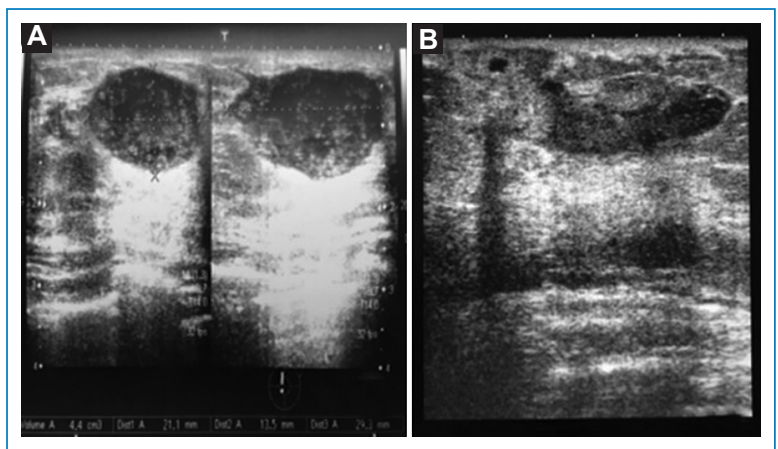

Figura 1. A: masa de bordes bien definidos, heterogénea de predominio hipoecoico con refuerzo acústico posterior. B: masa de bordes irregulares heterogénea hipoecoico con sombra acústica posterior con extensiones tubulares.
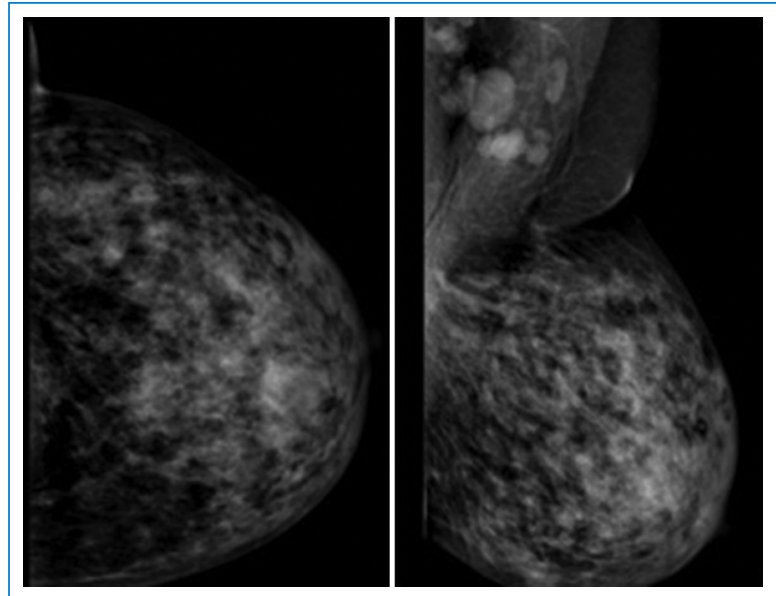

Figura 2. Asimetría focal en el cuadrante supero-interno de la mama izquierda, se realizó biopsia con diagnóstico de mastitis granulomatosa.

Los hallazgos mamográficos de mastitis granulomatosa encontrados en diferentes estudios son la asimetría de densidad del tejido mamario (focal o regional) o masa irregular, los hallazgos asociados son engrosamiento de la piel y adenopatías axilares ${ }^{10,13}$.

Según un estudio realizado por Barreto, et al. ${ }^{13}$ donde realizaron mamografía a 50 pacientes, el hallazgo mamográfico más frecuente fue una asimetría en el $42 \%$ de los casos, de estos la asimetría focal fue descrita en el $61,9 \%$ y la asimetría global en el 9,5\%. A su vez, se han informado ocasionalmente microcalcificaciones asociadas ${ }^{9}$.

Según un estudio realizado por Poyraz, et al. ${ }^{14}$, los hallazgos mamográficos más comunes incluyeron densidades asimétricas en siete pacientes (Breast Imaging

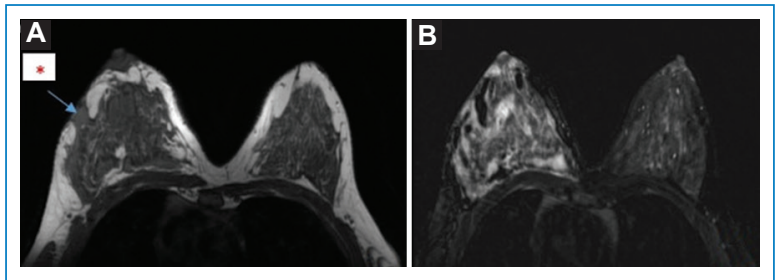

Figura 3. A: T1 mama derecha aumentada de tamaño con masa de márgenes irregulares que se extiende a la piel (*). B: T2 saturación grasa con hiperintensidad de la mama derecha.

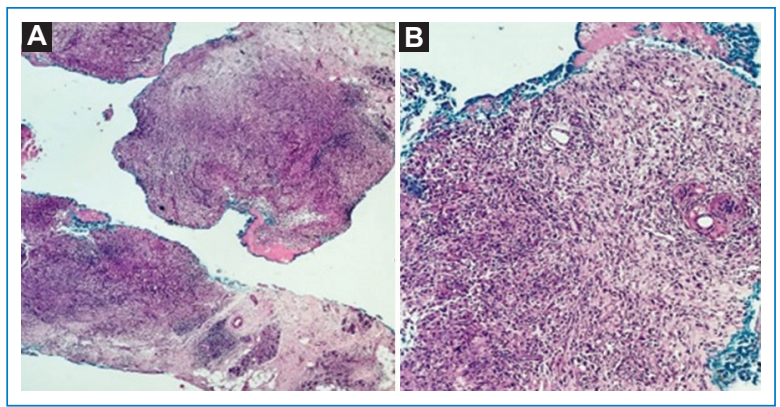

Figura 4. A: objetivo de $4 X$, hematoxilina-eosina. Se observan cilindros de parénquima mamario con un denso infiltrado inflamatorio que altera la arquitectura normal. B: aumento $10 \mathrm{X}$, infiltrado inflamatorio compuesto por linfocitos, plasmocitos, histiocitosis y células gigantes multinucleadas.

Reporting and Data System [BI-RADS] 0), masas circunscritas en tres (BI-RADS 3) y una masa mal definida en un paciente (BI-RADS 4). No se observaron microcalcificaciones en ninguno de los pacientes. Por otro lado, la ecografía mostró quistes complejos con paredes gruesas y septos con formación de fístulas en cuatro pacientes (BI-RADS 3), heterogeneidad parenquimatosa y obliteración de grasa subcutánea en seis pacientes (BI-RADS 0), y lesiones hipoecoicas heterogéneas irregulares en 10 pacientes (BI-RADS 4), lo cual ofrece un riesgo intermedio de malignidad que fue descartado con estudio histopatológico mediante biopsia, el método más confiable para el diagnóstico definitivo.

El tamaño de la lesión para una muestra adecuada debe ser de $2 \mathrm{~cm}$; la biopsia tru-cut debe realizarse guiada por imágenes. La sensibilidad y especificidad del tru-cut en comparación con la biopsia por aspiración con aguja fina deben considerarse, ya que la mastitis granulomatosa es un gran simulador del cáncer de mama y puede llevar a diagnósticos erróneos ${ }^{15}$. En nuestro estudio se realizó biopsia tru-cut teniendo 


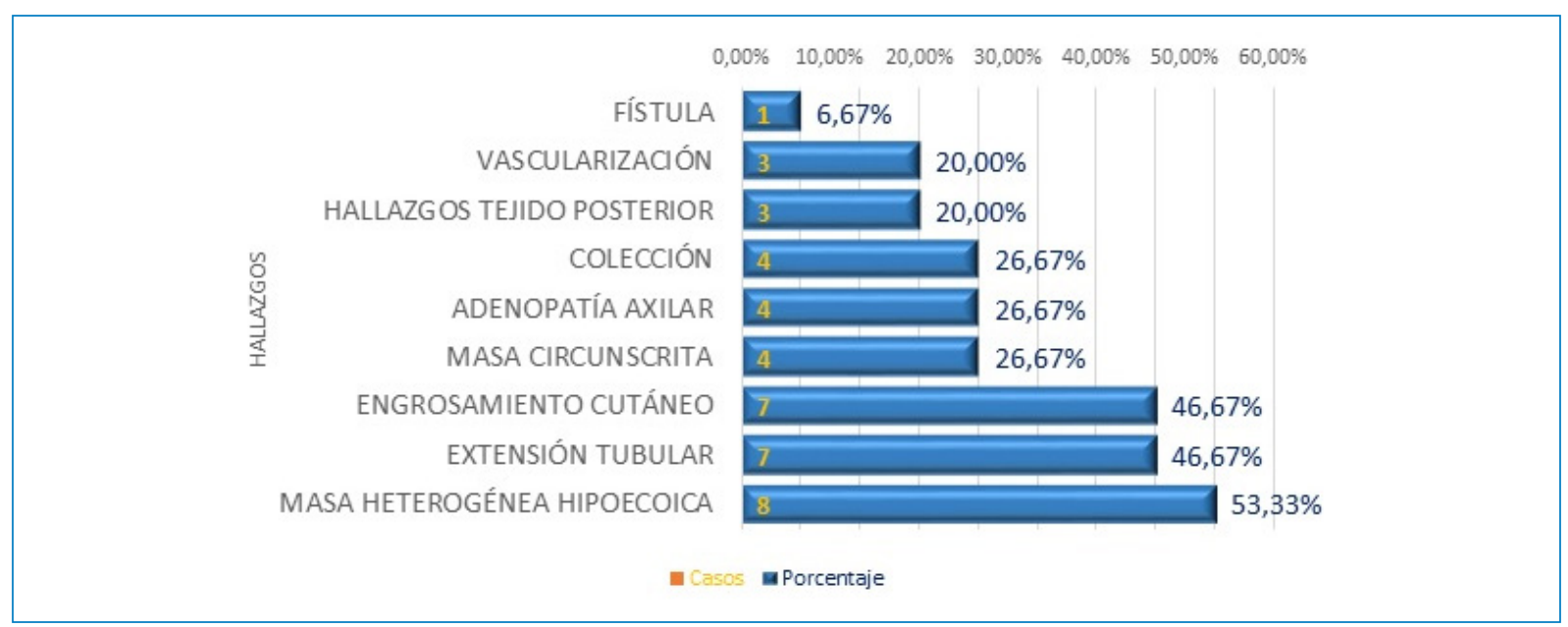

Figura 5. Hallazgos en ultrasonografía.

en cuenta su ventaja en la cantidad de material obtenido, y en la importancia que cumple esta característica en la diferenciación en cáncer de mama, además de ayudar a determinar si un cáncer de seno es invasivo o in situ ${ }^{13}$.

Los hallazgos varían, es más frecuente una gran masa hipoecoica heterogénea irregular $(53,33 \%)$ con múltiples extensiones tubulares, la mayoría de veces en eje paralelo a la piel; también se puede observar masas lobuladas o irregulares y/o masa circunscrita hipoecoica $(26,6 \%)^{1,16,17}$. Se han descrito el refuerzo acústico posterior y sombra acústica posterior ${ }^{1}$.

En la resonancia magnética se observan pequeñas lesiones que confluyen con bordes bien definidos, hiperintensas en secuencias de T2 o con realce en anillo que pueden representar microabscesos ${ }^{1,18}$. Si hay edema del parénquima este tiene señal hiperintensa en secuencias de T2 con curvas de realce de tipo I o tipo II (realce gradual y continuo o gradual y con meseta ${ }^{1,2}$.

El examen histológico de los lobulillos mamarios refleja una reacción inflamatoria granulomatosa, compuesta por histiocitos epitelioides y células gigantes multinucleadas, además de linfocitos, plasmocitos y, en ocasiones, leucocitos polinucleares, con ausencia de necrosis caseosa. Los cultivos para bacterias, la tinción de Ziehl-Neelsen y la tinción especial para hongos son negativas ${ }^{19}$.

El papel de las imágenes en la evaluación de la mastitis confirmada por biopsia es importante, se utiliza para establecer la ubicación de las lesiones, documentar el tamaño de las lesiones, identificar la formación de abscesos, evaluar respuesta al tratamiento e identificar enfermedad metacrónica y recurrencia local ${ }^{18}$.
Hay informes de casos de mastitis sintomática que enmascaran una neoplasia sincrónica que a menudo se ve como microcalcificaciones en mamografías obtenidas después de que los síntomas mamarios han resuelto; estos hallazgos apoyan la utilidad del seguimiento mamográfico después de la resolución de los síntomas que a menudo limitan la evaluación inicial con mamografía en mujeres en quienes está indicado. Aunque no hay consenso sobre el seguimiento radiológico, se ha sugerido mamografía anualmente y ecografía cada 3-6 meses después del episodio agudo ${ }^{11}$. En el estudio realizado solo se realizó mamografía a una paciente, sin embargo, se describe su importancia en el seguimiento de lesiones sintomáticas en el tejido mamario teniendo en cuenta que puede existir un enmascaramiento del cáncer de mama.

El diagnóstico diferencial de mayor importancia que excluir es el carcinoma inflamatorio, una forma agresiva de cáncer de seno que involucra invasión linfovascular. En mamografía del carcinoma inflamatorio presenta edema cutáneo extenso y engrosamiento trabecular, que son menos comunes en mastitis granulomatosa, mientras que la asimetría y distorsión arquitectónica se puede ver en ambas patologías ${ }^{11}$.

En general, la resonancia magnética del seno tiene una alta sensibilidad (91-100\%) en la detección de lesiones malignas. Está indicada cuando la evaluación con mamografía y ecografía son limitadas por edema parenquimatoso 0 densidad mamaria ${ }^{10,17}$.

Por otro lado, debido al reto radiológico que representa esta entidad, algunos autores han descrito el rendimiento diagnóstico de la ecografía vs. ecografía combinada con elastografía para distinguir entre mastitis 
y lesiones mamarias malignas; se ha encontrado que la sensibilidad, especificidad, valor predictivo positivo y valor predictivo negativo aumentan cuando se realizan ambas, con valores del $96,1,100,100,92,3$ y $97,3 \%$ respectivamente en comparación a la ecografía por sí sola, con valores del $94,8,66,7,85,9,85,7$ y $85,8 \%{ }^{17}$.

En conclusión, la mastitis granulomatosa es una enfermedad de difícil diagnóstico, con hallazgos clínicos y radiológicos inespecíficos que obligan a descartar patología neoplásica. El diagnóstico definitivo se realiza mediante el estudio histológico, sin embargo, la presentación clínica y radiológica son de vital importancia en la toma de decisiones clínicas y en la prevención de tratamientos erróneos.

\section{Financiamiento}

Los autores declaran la ausencia de fuentes de financiamiento.

\section{Conflicto de intereses}

Los autores declaran la ausencia de conflictos de intereses.

\section{Responsabilidades éticas}

Protección de personas y animales. Los autores declaran que para esta investigación no se han realizado experimentos en seres humanos ni en animales.

Confidencialidad de los datos. Los autores declaran que han seguido los protocolos de su centro de trabajo sobre la publicación de datos de pacientes.

Derecho a la privacidad y consentimiento informado. Los autores han obtenido el consentimiento informado de los pacientes y/o sujetos referidos en el artículo. Este documento obra en poder del autor de correspondencia.

\section{Bibliografía}

1. Cedric W, Nanyes JE, Quintero CJ, Mais DD, Kist KA, Dornbluth NC Idiopathic granulomatous mastitis: Manifestations at multimodality imaging and pitfalls. Radiographics. 2018;38(2):330-56.

2. Gautier N, Lalonde L, Tran-Thanh D, El Khoury M, David J, Labelle M, et al. Chronic granulomatous mastitis: Imaging, pathology and management. Eur J Radiol. 2013;82:e165-75.

3. Golobart A, Luna M, Madaula C, Mariscal A, Vizcaya S, Durany D, et al Mastitis granulomatosa: revisión de 4 casos diagnosticados y tratados en una unidad de patología mamaria. Rev Senologia Patol Mam. 2011;24(4):157-62.

4. Baslaim MM, Khayat $\mathrm{HA}, \mathrm{Al}$-Amoudi A. Idiopathic granulomatous mastitis: A heterogeneous disease with variable clinical presentation. World $\mathrm{J}$ Surg. 2007;31:1677-81.

5. Freeman CM, Xia BT, Wilson GC, Lewis JD, Khan S, Lee SJ, et al. Idiopathic granulomatous mastitis : A diagnostic and therapeutic challenge. Am J Surg. 2017;214:701-6.

6. Lester SC. Differential diagnosis of granulomatous mastitis. Breast J. 2005;11(6):534-5.

7. Oltean HN, Soliman AS, Omar OS, Youssef TF, Karkouri M, Abdel-Aziz A. Risk factors for chronic mastitis in morocco and egypt. Int J Inflam. 2013:2013:184921.

8. Sheybani F, Sarvghad M, Naderi H, Gharib M. Treatment for and clinical characteristics of granulomatous mastitis. Obstet Gynecol. 2015:125(4):801-7.

9. Handa P, Leibman AJ, Sun D, Abadi M, Goldberg A. Granulomatous mastitis: changing clinical and imaging features with image-guided biopsy correlation. Eur Radiol. 2014;24(10):2404-11.

10. Sripathi S, Ayachit A, Bala A, Kadavigere R, Kumar S. Idiopathic granuIomatous mastitis: a diagnostic dilemma for the breast radiologist. Insights Imaging. 2016;7(4):523-9.

11. Pluguez-Turull CW, Nanyes JE, Quintero CJ, Alizai H, Mais DD, Kist KA, et al. Idiopathic granulomatous mastitis: manifestations at multimodality imaging and pitfalls. Radiographics. 2018;38(2):330-56.

12. Dursun M, Yilmaz S, Yahyayev A, Salmaslioglu A, Yavuz E, Igci A, et al. Multimodality imaging features of idiopathic granulomatous mastitis: outcome of 12 years of experience. Radiol Med. 2012;117(4): 529-38.

13. Barreto DS, Sedgwick EL, Nagi CS, Benveniste AP. Granulomatous mastitis: etiology, imaging, pathology, treatment, and clinical findings. Breast Cancer Res Treat. 2018;171(3):527-34.

14. Poiyraz N, Emilik G, Batur A, Gundes E, Keskin S. Magnetic resonance imaging features of idiopathic granulomatous mastitis: a retrospective analysis. Iran J Radiol. 2016;13(3):e20873.

15. Guio Ávila Jl, Cruz Rueda AR, Pérez Morón JE. Mastitis granulomatosa: presentación clínica, imagenológica e histológica. Serie de casos. Repert Med Cir. 2016;25(4):235-40.

16. Larsen LJH, Klipfel N, Grant E, lyengar G. Granulomatous lobular mastitis: Imaging, diagnosis, and treatment. AJR Am J Roentgenol. 2009;193(2):574-81

17. Arslan S, Öncü F, Eryılmaz MA, Durmaz MS, Altunkeser A, Ünlü Y. Advantages of b-mode ultrasound combined with strain elastography in differentiation of idiopathic granulomatous mastitis from malignant breast lesions. Turk J Med Sci. 2018;48(1):16-23.

18. Fazzio RT, Shah SS, Sandhu NP, Glazebrook KN. Idiopathic granulomatous mastitis: imaging update and review. Insights Imaging. 2016; 7(4):531-9.

19. Lai ECH, Chan WC, Ma TKF, Tang APY, Poon CSP. The role of conservative treatment in idiopathic granulomatous mastitis. Breast $\mathrm{J}$. 2005;11(6):454-6. 